\title{
Seletividade de inseticidas a Doru luteipes (Scudder, 1876) (Dermaptera: Forficulidae) e Cotesia sp. (Hymenoptera: Braconidae) inimigos naturais de Ascia monuste orseis (Godart, 1818) (Lepdoptera: Pieridae)
}

\author{
Selectivity of insecticides to Doru luteipes (Scudder, 1876) (Dermaptera: Forficulidae) and \\ Cotesia sp. (Hymenoptera: Braconidae) natural enemies of Ascia monuste orseis (Godart, \\ 1818) (Lepdoptera: Pieridae)
}

\section{Marcelo Coutitnho Picanço ${ }^{1}$ Marcelo Fialho de Moura ${ }^{2}$ Moacyr Mascarenhas Motta Miranda ${ }^{3}$ Lessando Moreira Gontijo ${ }^{4}$ Flávio Lemes Fernandes ${ }^{4}$}

\section{RESUMO}

Este trabalho objetivou estudar a seletividade dos inseticidas carbaril, deltametrina, paratiom metílico, permetrina e triclorfom em favor do predador Doru luteipes (Scudder) (Dermaptera: Forficulidae) e do parasitóide Cotesia sp. (Hymenoptera: Braconidae), em relação ao controle do curuquerê Ascia monuste orseis (Godart) (Lepidoptera: Pieridae). Foram determinadas as CL90 para A. monuste orseis e estas foram utilizadas como doses discriminatórias para se avaliar o grau de seletividade dos inseticidas. Deltametrina e permetrina foram altamente seletivos em favor de D. luteipes. Deltametrina foi medianamente seletiva em favor de Cotesia sp. e a permetrina não apresentou seletividade em favor deste parasitóide. Triclorfom foi altamente seletivo em favor de ninfas de quarto estádio e adultos de D. luteipes e mediamente seletivo em favor de Cotesia sp. e de ninfas de primeiro estádio do predador. Carbaril foi medianamente seletivo em favor de Cotesia sp. mas não apresentou seletividade em favor de $\boldsymbol{D}$. luteipes. Paratiom metílico não foi seletivo em favor de Cotesia sp. e D. luteipes. Adultos de D. luteipes foram menos sensiveis a deltametrina, permetrina e triclorfom do que adultos de Cotesia sp., sendo que o inverso ocorreu com o carbaril. Esses inimigos naturais apresentaram alta sensibilidade ao paratiom metílico. Ninfas de quarto estádio e adultos de D. luteipes foram mais sensiveis ao paratiom metílico e triclorfom que ninfas de primeiro estádio. Ninfas e adultos de D. luteipes apresentaram semelhante sensibilidade ao carbaril, deltametrina e permetrina.

Palavras-chave: Brássicas, Braconidae, manejo integrado de pragas, inimigos naturais, curuquerê da couve.

\section{ABSTRACT}

This work aimed to study the selectivity of the insecticides carbaryl, deltamethrin, methyl parathion, permethrin and trichlorfon in favor of the predator Doru luteipes (Scudder) (Dermaptera: Forficulidae) and of the parasitoid Cotesia sp. (Hymenoptera; Braconidae) in relation to the control of the kale leafworm Ascia monuste orseis (Godart) (Lepidoptera: Pieridae). $L C_{90}$ were determined for $A$. monuste orseis, and these were used as discriminatory doses to evaluate the degree of selectivity of the insecticides. Deltamethrin and permethrin were highly selective in favor of D. luteipes. Deltamethrin was intermediate selectivity in favor of Cotesia sp., while permethrin was not selective in favor of this parasitoid. Trichlorfon was highly selective in favor of fourth instar nymphs and adults of $\boldsymbol{D}$. luteipes, and it was intermediate selectivity in favor of Cotesia sp. and first instar nymphs of the predator. Carbaryl was intermediate selectivity in favor of Cotesia sp., but it did not show selectivity in favor of D. luteipes. Methyl parathion did not show selectivity in favor of D. luteipes and

${ }^{1}$ Professor Adjunto, Doutor, Departamento de Biologia Animal, Universidade Federal de Viçosa (UFV), 36571-000, Viçosa, MG. E-mail: picanco@mail.ufv.br. Autor para correspondência.

${ }^{2}$ Engenheiro Agrônomo, doutorando do Programa de Pós-graduação em Entomologia.

${ }^{3}$ Engenheiro Agrônomo, Mestre, Departamento de Biologia Animal, UFV.

${ }^{4}$ Engenheiro Agrônomo, Departamento de Biologia Animal, UFV. 
Cotesia sp. Adults of D. luteipes were less sensitive to deltamethrin, permethrin and trichlorfon than adults of Cotesia sp. and the inverse happened with carbaryl. Both natural enemies presented high sensibility to methyl parathion. Fourth instar nymphs of and adults of $\boldsymbol{D}$. luteipes were more sensitive to methyl parathion and trichlorfon than first instar nymphs. Both nymphs and adults of D. luteipes presented similar sensibility to the carbaryl, deltamethrin and permethrin.

Key words: Brassica, Braconidae, integrated pest management, natural enemies, kale leafworm.

\section{INTRODUÇÃO}

O curuquerê Ascia monuste orseis (Godart) (Lepidoptera: Pieridae) constitui uma das principais pragas de brássicas no Brasil, por ocasionar intensa desfolha às plantas.

Em função do ataque de pragas os agricultores adotam medidas de controle, as quais devem ser selecionadas de forma a se buscar a sustentabilidade ambiental e econômica. Tais sustentabilidades são favorecidas pela adoção de táticas de controle pouco danosas ao ambiente, como o uso de plantas resistentes, a adoção do controle biológico e de práticas culturais. Porém, a maioria desses procedimentos são preventivos, e em situações de ataque severo o agricultor lança mão de medidas terapêuticas, dentre as quais a mais amplamente difundida e adotada é o controle químico. Logo, é de fundamental importância selecionar inseticidas eficientes no controle da praga e seletivos em favor de seus inimigos naturais (BECKER et al., 1989, KOVACH et al., 1992).

Existem dois tipos de seletividade de inseticidas: a ecológica (que é obtida pela metodologia de aplicação do inseticida de forma a minimizar a exposição do inimigo natural a este) e a fisiológica (que é obtida pela aplicação de inseticidas mais tóxicos à praga que aos inimigos naturais).

Entre os inimigos naturais dessa praga, estão o predador Doru luteipes (Scudder) (Dermaptera: Forficulidae) e o parasitóide Cotesia sp. (Hymenoptera: Braconidae) (PENTEADO-DIAS, 1986, GOBBI et al., 1989 e 1990, RENESTO \& TERADA, 1992, CAVALCANTE et al., 1996). Entre os principais inseticidas utilizados no controle de $A$. monuste orseis em brássicas, estão o carbaril, a deltametrina, o paratiom metílico, a permetrina e o triclorfom (CRESPO et al., 2001). Apesar do curuquerê da couve ser uma praga de registro antigo (SILVA et al., 1968) e de sua importância como praga em culturas de brássicas, pouco se conhece sobre o grau de seletividade fisiológica desses inseticidas aos seus inimigos naturais (CRESPO et al., 2001).
Buscando-se suprir essa carência de informação, este trabalho teve por objetivo estudar a seletividade fisiológica dos inseticidas carbaril, deltametrina, paratiom metílico, permetrina e triclorfom ao predador D. luteipes e ao parasitóide Cotesia sp. em relação ao controle de $\boldsymbol{A}$. monuste orseis.

\section{MATERIAL EMÉTODOS}

Este trabalho foi conduzido no laboratório de Manejo Integrado de Pragas da Universidade Federal de Viçosa (UFV). Os insetos utilizados nos bioensaios foram coletados no Campus da UFV. Os inseticidas foram escolhidos de forma a abranger os principais produtos usados no controle de $\boldsymbol{A}$. monuste orseis em brássicas, selecionando-se para tanto, os piretróides deltametrina $25 \mathrm{CE}$ e permetrina $500 \mathrm{CE}$, o clorofosforado triclorfom $500 \mathrm{SC}$, o carbamato carbaril 850 PM e o organofosforado paratiom metílico $600 \mathrm{CE}$. Foram realizados dois bioensaios e nestes o delineamento experimental foi inteiramente casualizado com quatro repetições. Cada parcela experimental foi constituída por placa de Petri contendo 10 insetos.

No primeiro bioensaio, determinaram-se as concentrações dos inseticidas que causavam $90 \%$ de mortalidade ( $\mathrm{CL}_{90}$ ) a lagartas de $\boldsymbol{A}$. monuste orseis. Para instalação deste bioensaio, folhas de couve do clone Portuguesa foram imersas em caldas inseticidas e em água (testemunha) por cinco segundos. Em todos os tratamentos empregou-se espalhante adesivo NDodecil benzeno sulfonato de sódio $320 \mathrm{CE}$ na dosagem de 0,096 $\mathrm{mg}$ de ingrediente ativo/mL de calda (ANDREI, 1996). As folhas foram mantidas por duas horas em casa de vegetação com circulação de ar para retirar o excesso de umidade e, posteriormente, foram acondicionadas em placas de Petri de $9 \mathrm{~cm}$ de diâmetro por $2 \mathrm{~cm}$ de altura. Em cada placa de Petri, foram colocadas 10 lagartas de quinto ínstar de $\boldsymbol{A}$. monuste orseis. As placas de Petri foram levadas para estufa incubadora a $25 \pm 0,5^{\circ} \mathrm{C}$ e umidade relativa de $75 \pm 5 \%$. Vinte e quatro horas após avaliou-se a mortalidade dos insetos, sendo que esta foi corrigida em relação à ocorrida na testemunha, utilizando-se a fórmula de ABBOTT (1925). Por análise de próbite, determinaramse curvas de concentração-mortalidade dos inseticidas para $\boldsymbol{A}$. monuste orseis. Foram aceitas curvas cuja probabilidade de aceitação da hipótese de nulidade (de que os dados possuem distribuição de próbite) pelo teste $\chi^{2}$ fosse maior que 0,05 (YOUNG \& YOUNG, 1998). Por meio destas curvas, foram estimadas as $\mathrm{CL}_{90}$ dos inseticidas para as lagartas de $\boldsymbol{A}$. monuste orseis.

No segundo bioensaio, adultos e ninfas de primeiro e quarto estádio do predador D. luteipes e 
adultos do parasitóide Cotesia sp. foram submetidos às $\mathrm{CL}_{90}$ determinadas para $\boldsymbol{A}$. monuste orseis. Para exposição dos inimigos naturais aos inseticidas, foi adotado procedimento semelhante ao do primeiro bioensaio. Os valores de mortalidade dos inimigos naturais foram transformados em, arco-seno $\sqrt{\frac{x}{100}}$,para realização de análise de variância e comparação das médias pelo teste de Scott-Knott a 5\% de significância.

\section{RESULTADOS E DISCUSSÃO}

A deltametrina foi o inseticida que apresentou curva concentração-mortalidade para $\boldsymbol{A}$. monuste orseis com maior inclinação. Já a permetrina apresentou a curva concentração-mortalidade com a menor inclinação para este inseto-praga (Tabela 1). Portanto, a permetrina foi o inseticida que apresentou maior segurança na obtenção de controle satisfatório deste inseto-praga em condições de má calibração dos pulverizadores, uma vez que grandes variações em sua dose provocarão pequenas variações na mortalidade de $A$. monuste orseis.

A deltametrina e a permetrina apresentaram os menores valores de concentrações letais (Tabela 1), sendo portanto os inseticidas de maior potência de controle de $\boldsymbol{A}$. monuste orseis. Segundo NAUMANN (1990), a alta potência dos piretróides está associada a sua alta taxa de penetração no corpo de inseto por diversas rotas e ao seu mecanismo de ação (interferem na abertura e conformação dos canais de $\mathrm{Na}^{+}$dos neurônios).

As concentrações de deltametrina e permetrina que causaram $90 \%$ de mortalidade a $\boldsymbol{A}$. monuste orseis ocasionaram 63,30; 2,50; 19,17 e 2,27\% e 73,$04 ; 0,00 ; 21,12$ e $3,57 \%$ de mortalidade ao

Tabela 1 - Equações e probabilidade das curvas concentração - mortalidade e $\mathrm{CL}_{90}$ (mg de ingrediente ativo/mL) de cinco inseticidas para lagartas de Ascia monuste orseis.

\begin{tabular}{lccc}
\hline Inseticidas & Equações $^{1 /}$ & Probabilidade & CL $_{90}\left(\mathrm{IC}_{95 \%}\right)^{2 /}$ \\
\hline Carbaril & $\mathrm{y}=6,82+3,22 \mathrm{x}$ & 0,309 & $\begin{array}{c}0,6797 \\
(0,5836-0,8284) \\
0,0052\end{array}$ \\
Deltametrina & $\mathrm{y}=19,96+6,00 \mathrm{x}$ & 0,500 & $(0,0047-0,0061)$ \\
Paratiom Metílico & $\mathrm{y}=13,02+4,93 \mathrm{x}$ & 0,293 & $\left.\begin{array}{c}0,0429 \\
(0,0382-0,0499) \\
0,0183\end{array}\right)$ \\
Permetrina & $\mathrm{y}=9,65+1,94 \mathrm{x}$ & 0,100 & $(0,0134-0,0286)$ \\
0,1287 \\
Triclorfom
\end{tabular}

1/ $\mathrm{y}=$ mortalidade (próbite) e $\mathrm{x}=$ logaritmo da concentração (mg do ingrediente ativo/mL).

${ }^{2 /} \mathrm{IC}_{95 \%}=$ Intervalo de confiança a $95 \%$ de probabilidade. parasitóide Cotesia sp., adultos, ninfas de primeiro e quarto estádio do predador $\boldsymbol{D}$. luteipes, respectivamente (Tabela 2). Portanto, os piretróides deltametrina e permetrina foram altamente seletivos em favor de todos os estádios de desenvolvimento do predador. A deltametrina foi medianamente seletiva em favor de Cotesia sp. e a permetrina não apresentou seletividade a adultos deste parasitóide.

Segundo YU (1987 e 1988), a seletividade dos piretróides em favor de inimigos naturais está associada à menor taxa de penetração dessas moléculas na cutícula do inimigo natural, menor taxa de metabolização dessas por oxidases microssomais e esterases e alteração do alvo de ação desses princípios ativos, gerando menor efeito tóxico destes inseticidas nos inimigos naturais do que em suas presas ou hospedeiros. A maior seletividade da deltametrina em relação à permetrina em favor de Cotesia sp., provavelmente seja devido ao maior peso molecular da deltametrina $(505,2)$ do que o da permetrina $(391,3)$, já que substâncias com maiores pesos moleculares possuem menor taxa de penetração na cutícula dos insetos (STOCK \& HOLLOWAY, 1993).

Resultados semelhantes aos observados para Cotesia sp. com a deltametrina neste trabalho foram obtidos por MANI (1995) para adultos de Cotesia plutellae (Kurdjumov) e THAKUR \& DEKA (1995) para Cotesia glomerata (L.). Entretanto, OBRA \& MORALLO (1997) e TALEKAR \& YANG (1991) verificaram alta toxicidade deste inseticida a esses parasitóides. Como observado neste trabalho para $\boldsymbol{D}$. luteipes em relação a $\boldsymbol{A}$. monuste orseis, REIS et al. (1988) e FALEIRO et al. (1995) também verificaram seletividade de deltametrina e permetrina em favor deste predador em relação à sua presa Spodoptera frugiperda (Smith) (Lepidoptera: Noctuidae), sendo que o mesmo foi observado por SIMÕES et al. (1998) para deltametrina.

A concentração de triclorfom que causou $90 \%$ de mortalidade a $\boldsymbol{A}$. monuste orseis foi altamente seletiva em favor de ninfas de quarto estádio e adultos de $\boldsymbol{D}$. luteipes e mediamente seletiva em favor de Cotesia sp. e ninfas de primeiro estádio do predador (Tabela 2). A seletividade do triclorfom em favor de Cotesia sp. e D. luteipes, possivelmente esteja associada a sua alta solubilidade em água (12\%) (MEISTER, 2001), o que faz com que esta molécula tenha baixa taxa de penetração na cutícula destes insetos que é composta principalmente de compostos lipídicos (LEITE et al., 1998). PICANÇO et 
Tabela 2 - Mortalidades (\%) de adultos do parasitóide Cotesia sp. e de adultos e ninfas de primeiro e quarto estádio do predador Doru luteipes causadas pelas $\mathrm{CL}_{90}$ de cinco inseticidas a lagartas de Ascia monuste orseis.

\begin{tabular}{|c|c|c|c|}
\hline \multirow[t]{2}{*}{ Inseticidas } & \multicolumn{3}{|c|}{ Mortalidade $(\%)^{1 /}$} \\
\hline & \multicolumn{2}{|c|}{ Adultos de D. luteipes } & Adultos de Cotesia sp. \\
\hline Paratiom metílico & \multicolumn{2}{|c|}{$72,50 \mathrm{aB}$} & $96,03 \mathrm{aA}$ \\
\hline Carbaril & \multicolumn{2}{|l|}{$100,00 \mathrm{aA}$} & $50,96 \mathrm{bB}$ \\
\hline Permetrina & \multicolumn{2}{|l|}{$0,00 \mathrm{bC}$} & $73,04 \mathrm{aA}$ \\
\hline Deltametrina & \multicolumn{2}{|l|}{$2,50 \mathrm{bC}$} & $63,30 \mathrm{aB}$ \\
\hline Triclorfom & \multicolumn{2}{|l|}{$0,00 \mathrm{bC}$} & $34,19 \mathrm{aB}$ \\
\hline \multirow[t]{2}{*}{ Inseticidas } & \multicolumn{3}{|c|}{ Mortalidade $(\%)^{1 /}$} \\
\hline & Ninfa I de $\boldsymbol{D}$. luteipes & Ninfa IV de D. luteipes & Adulto de $\boldsymbol{D}$. luteipes \\
\hline Carbaril & $100,00 \mathrm{aA}$ & $92,50 \mathrm{aA}$ & $100,00 \mathrm{aA}$ \\
\hline Paratiom metílico & $100,00 \mathrm{aA}$ & $72,50 \mathrm{bA}$ & $72,50 \mathrm{bB}$ \\
\hline Triclorfom & $53,69 \mathrm{aB}$ & $5,00 \mathrm{bB}$ & $0,00 \mathrm{bC}$ \\
\hline Permetrina & $21,12 \mathrm{aC}$ & $3,57 \mathrm{aB}$ & $0,00 \mathrm{aC}$ \\
\hline Deltametrina & $19,17 \mathrm{aC}$ & $2,27 \mathrm{aB}$ & $2,50 \mathrm{aC}$ \\
\hline
\end{tabular}

${ }^{1 /}$ As médias seguidas pela mesma letra minúscula na linha ou maiúscula na coluna não diferem, entre si, pelo teste de Scott-Knott a 5\% de probabilidade do erro.

al. (1998) também observaram que o triclorfom é seletivo em favor do predador Polybia ignobilis (Haliday) (Hymenoptera: Vespidae) em relação à sua presa $\boldsymbol{A}$. monuste orseis.

A concentração do carbamato carbaril que causou $90 \%$ de mortalidade a $\boldsymbol{A}$. monuste orseis foi medianamente seletiva em favor de Cotesia sp. e não seletiva em favor de nenhum dos estádios de desenvolvimento do predador (Tabela 2).

A seletividade do carbamato carbaril em favor de Cotesia sp, possivelmente, esteja associada a alterações na enzima acetilcolinesterase no corpo desse parasitóide ou a maior velocidade com que a enzima acetilcolinesterase catalisa a hidrólise do neurotransmissor acetilcolina no parasitóide do que nas lagartas (SILVER et al., 1995). A seletividade desse carbamato também pode estar associada à maior taxa de metabolização desse pelo parasitóide do que pela praga por enzimas monooxigenases dependentes de citocromo P450 (BRATTSTEN et al., 1986). Resultados semelhantes aos observados com Cotesia sp. para o carbaril foram verificados por MANI (1995) para $\boldsymbol{C}$. plutellae e TALEKAR \& YANG (1991) e THAKUR \& DEKA (1995) para $\boldsymbol{C}$. glomerata.

A concentração do paratiom metílico que causou $90 \%$ de mortalidade a $\boldsymbol{A}$. monuste orseis não foi seletiva em favor do parasitóide Cotesia sp. e a nenhum dos estádios de desenvolvimento do predador D. luteipes (Tabela 2). Também PICANÇO et al. (1998) observaram que o paratiom metílico não apresentou seletividade em favor do predador $\boldsymbol{P}$. ignobilis em relação a sua presa $\boldsymbol{A}$. monuste orseis. Entretanto, TILLMAN \& SCOTT (1997) verificaram baixa toxicidade do paratiom metílico ao parasitóide Cotesia marginiventris $\left(\begin{array}{lllllll}\mathrm{C} & \mathrm{r} & \mathrm{e} & \mathrm{s} & \mathrm{s} & \mathrm{o} & \mathrm{n}\end{array}\right)$ ( $\mathrm{H}$ y m e n o p te ra: Braconidae).

Os adultos do predador D. luteipes foram menos sensíveis a deltametrina, permetrina e triclorfom em relação aos adultos do parasitóide Cotesia sp., sendo que o inverso ocorreu com o carbaril. Essas espécies apresentaram na fase adulta semelhante sensibilidade ao paratiom metílico (Tabela 2). As ninfas de quarto estádio e adultos do predador $\boldsymbol{D}$.

luteipes foram mais sensíveis ao paratiom metílico e triclorfom que suas ninfas de primeiro estádio. FALEIRO et al. (1995) observaram que os adultos de $\boldsymbol{D}$. luteipes foram mais tolerantes aos inseticidas carbaril, deltametrina e permetrina que ninfas deste predador. Ninfas e adultos de D. luteipes apresentaram semelhante sensibilidade ao carbaril, deltametrina e permetrina (Tabela 2).

\section{CONCLUSÕES}

Deltametrina e permetrina foram altamente seletivos em favor de D. luteipes. Deltametrina foi medianamente seletiva em favor de Cotesia sp. e a permetrina não apresentou seletividade em favor deste parasitóide.

Triclorfom foi altamente seletivo em favor de ninfas de quarto estádio e de adultos de D. luteipes e mediamente seletivo em favor de Cotesia sp. e de ninfas de primeiro estádio do predador.

Carbaril foi medianamente seletivo em favor de Cotesia sp., mas não foi seletivo em favor de $\boldsymbol{D}$. luteipes. Paratiom metílico não foi seletivo em favor de Cotesia sp. e de D. luteipes.

Adultos de D. luteipes foram menos sensíveis à deltametrina, permetrina e triclorfom do que adultos de Cotesia sp., sendo que o inverso ocorreu com o carbaril. Estes inimigos naturais apresentaram semelhante sensibilidade ao paratiom metílico.

Ninfas de quarto estádio e adultos de $\boldsymbol{D}$. luteipes foram mais tolerantes ao paratiom metílico e triclorfom que ninfas de primeiro estádio. Ninfas e adultos de D. luteipes apresentaram semelhante sensibilidade ao carbaril, deltametrina e permetrina. 


\section{AGRADECIMENTOS}

À Fundação de Amparo a Pesquisa de Minas Gerais (FAPEMIG) e ao CNPq pelo financiamento do projeto e pelas bolsas concedidas e à Profa. Angélica Maria Penteado-Dias da UFSCAR devido à identificação do parasitóide Cotesia sp.

\section{REFERÊNCIAS BIBLIOGRÁFICAS}

ABBOTT, W.S. A method of computing the effectiveness of an insecticide. Journal of Economic Entomology, Lanham, v.18, n.1, p.265-267, 1925.

ANDREI, E. Compêndio de defensivos agrícolas. 5.ed. São Paulo : Andrei, 1996. 506p.

BECKER, R.L. et al. Pesticides: Surface runoff, leaching, and exposure concerns. St. Paul : Minnesota Extension Service Publication, 1989. 15p. (Boletim Técnico, AG-BU3911).

BRATTSTEN, L.B. et al. Insecticide resistance: Challenge to pest management and basic research. Science, New York, v.231, n.4743, p.1255-1260, 1986.

CAVAlCANTE, T.R.M. et al. Controle natural de Ascia monuste orseis (Lepidoptera: Pieridae) em couve comum, Foz do Iguaçu, PR, 1996. In: SICONBIOL, 5., 1992, Foz do Iguaçu. Resumos... Foz do Iguaçu : EMBRAPA/CNPSo, 1996. p.429.

CRESPO, A.L.B. et al. Seletividade fisiológica de inseticidas a Vespidae predadores de Ascia monuste orseis (Lepidoptera: Pieridae) Pesquisa Agropecuária Brasileira, Brasília, v.16, n.11, p.2130-2136, 2001.

FALEIRO, F.G. et al. Seletividade de inseticidas a Spodoptera frugiperda (J.E. Smith) (Lepidoptera: Noctuidae) e o predador Doru luteipes (Scudder) (Dermaptera: Forficulidae). Anais da Sociedade Entomológica do Brasil, Londrina, v.24, n.2, p.247-252, 1995.

GOBBI, N. et al. Efeito do parasitismo de Cotesia glomerata (Linnaeus, 1758) (Hymenoptera, Braconidae) no consumo alimentar de lagartas de Ascia monuste orseis (Godart, 1818) (Lepidoptera, Pieridae). Anais da Sociedade Entomológica do Brasil, Porto Alegre, v.18, n.1, p.169-175, 1989.

GOBBI, N. et al. Oviposition pattern by Cotesia ayerzai (Hym.: Braconidae) on Ascia monuste orseis (Lep.: Pieridae) under laboratory conditions. Entomophaga, Paris, v.35, n.2, p.195202, 1990 .

KOVACH, J.C. et al. A method to measure the environmental impact of pesticides. Ithaca, New York : Cornell University, 1992. 16p. (New York's Food and Life Sciences Bulletin, 139).

LEITE, G.L.D. et al. Selectivity of insecticides with and without mineral oil to Brachygastra lecheguana (Hymenoptera: Vespidae), a predator of Tuta absoluta (Lepidoptera: Gelechiidae). Ceiba, Tegucigalpa, v.39, n.1, p.3-6, 1998.

MANI, M. Studies on the toxicity of pesticides to Cotesia plutellae (Hymenoptera: Braconidae), a parasitoid of diamondback moth, Plutella xylostella (L.). Journal of Insect Science, Tucson, v.8, n.1, p.31-33, 1995.

MEISTER. Farm chemicals handbook. Willoughby : Meister, 2001. 1000p.

NAUMANN, K. Synthetic pyrethroid insecticides: structures and properties. Berlin : Springer-Verlag, 1990. $241 \mathrm{p}$.

OBRA, J.B.; MORALLO, R.B. Sensitivity of Cotesia plutellae and Diadegma semiclausum, parasitoids of diamondback moth, Plutella xylostella (L.) to several insecticides. Philippine Entomologist, Laguna, v.11, n.1, p.49-56, 1997.

PENTEADO-DIAS, A.M. Parasitismo de Ascia monuste orseis (Latreille, 1819) (Lepidoptera: Pieridae) por Cotesia glomerata (L., 1758) (Hymenoptera: Braconidae: Microgastrinae). Revista Brasileira de Entomologia, São Paulo, v.30, n.2, p.257-259, 1986.

PICANÇO, M.C. et al. Seletividade de inseticidas a Polybia ignobilis (Haliday) (Hymenoptera: Vespidae) predador de Ascia monuste orseis (Godart) (Lepidoptera: Pieridae). Anais da Sociedade Entomológica do Brasil, Londrina, v.27, n.1, p.85-90, 1998.

REIS, L.L.; OLIVEIRA, L.J.; CRUZ, I. Biologia e potencial de Doru luteipes no controle de Spodoptera frugiperda. Pesquisa Agropecuária Brasileira, Brasília, v.23, n.4, p.333-342, 1988 .

RENESTO, E.; TERADA, Y. Relationship between the parasitoid Apanteles ayerzai (Brethes, 1920) (Hymenoptera: Braconidae) and Ascia monuste orseis (Godart, 1819) (Lepidoptera: Pieridae): effects of host age. Ciência e Cultura, São Paulo, n.42, v.11, p.967970,1992 .

SILVA, A.G. et al. Quarto catálogo das plantas que vivem nas plantas do Brasil, seus parasitas e predadores. Rio de Janeiro : Ministério da Agricultura, 1968. 906p.

SILVER, A.R.J.; VAN EMDEN, H.F.; BATTERSBY, M. A biochemical mechanism of resistance to pirimicarb in two glasshouse clones of Aphis gossypii. Pesticide Science, London, v.43, n.1, p.21-29, 1995.

SIMÕES, J.C.; CRUZ, I.; SALGADO, L.O. Seletividade de inseticidas às diferentes fases de desenvolvimento do predador Doru luteipes (Scudder) (Dermaptera: Forficulidae). Anais da Sociedade Entomológica do Brasil, Londrina, v.27, n.2, p.289-294, 1998.

STOCK, D.; HOLLOWAY, P.J. Possible mechanisms for surfactant-induced foliar uptake of agrochemicals. Pesticide Science, London, v.38, n.2/3, p.165-177, 1993.

TALEKAR, N.S.; YANG, J.C. Characteristic of parasitism of diamondback moth by two larval parasites. Entomophaga, Paris, v.36, n.1, p.95-104, 1991.

THAKUR, N.S.A.; DEKA, T.C. Evaluation of insecticides for safety to Apanteles glomeratus (L.), a parasitoid of Pieris brassicae (L.). Pest Management in Horticultural Ecosystems, Bangalore, v.1, n.1, p.21-25, 1995. 
TILlMAN, P.G.; SCOTT, W. Susceptibility of Cotesia marginiventris (Cresson) (Hymenoptera: Braconidae) to field rates of selected cotton insecticides. Journal of Entomological Science, Athens, v.32, n.3, p.303-310, 1997.

YOUNG, L.J.; YOUNG, J.H. Statistical ecology - A population perspective. Boston: Kluwer, 1998. $565 \mathrm{p}$.
YU, S.J. Biochemical defense capacity in the spined soldier bug (Podisus maculiventris) and its lepidopterous prey. Pesticide Biochemical Physiology, San Diego, v.28, n.3, p.216-223, 1987.

YU, S.J. Selectivity of insecticides to the spined bug (Heteroptera: Pentatomidae) and its lepidopterous prey. Journal of Economic Entomology, Lanham, v.81, n.1, p.119-122, 1988. 